\title{
Factores pronóstico de las variantes de cáncer papilar de tiroides*
}

\author{
Drs. OSVALDO IRIBARREN B..$^{1,2}$, JUAN MADARIAGA G. ${ }^{2,3}$, VITORIO ZAFFIRI M. ${ }^{2,3}$, \\ PATRICIO HUIDOBRO M. ${ }^{4}$, Als. MAXIMILIANO MERTENS P. ${ }^{5}$, GABRIEL IRIBARREN R. ${ }^{5}$
}

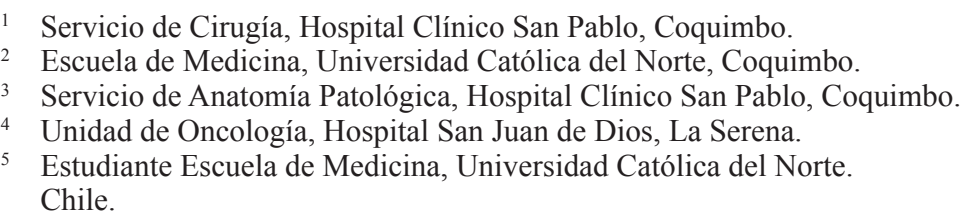

\begin{abstract}
Association between pathological variants of papillary thyroid cancer and prognosis
\end{abstract}

Background: Papillary thyroid carcinoma had a survival over 90\% in stages I , II and III. Treatment failures are observed in advanced tumors, when there is lymph node involvement or when there is vascular invasion. Aim: To assess the prognostic value of pathological variants of papillary thyroid carcinoma. Material and Methods: Review of pathological records of patients with papillary thyroid cancer operated between January 1999 and December 2008 in a regional hospital. The pathological variant of the tumor was revised and classified again as follicular variant, sclerosing, solid, tall cell variant, columnar variant, oncocytic, cribriform, microcarcinoma, insular variant and poorly differentiated. Results: The records of 51 females aged $48 \pm 16$ years and 12 males aged $42 \pm 18$ years were identified. Forty patients had a well differentiated carcinoma $(63 \%), 16$ had a follicular variant $(25 \%)$, four had a sclerosing variant and three other types. Capsule involvement was observed in six of 27 stage I, four of nine stage II, 20 of 26 stage III and one of one stage four tumor. Peritumoral lymphatic invasion was observed in 12 of 27 stage I, four of nine stage II, 21 of 26 stage III and one of one stage IV tumor. Lymph node relapse was observed in three patients without and nine patients with capsule involvement $(\mathrm{p}<0.01)$ and in 11 of 38 patients without and 27 of 38 patients with lymphatic invasion $(\mathrm{p}<0.01)$. Two patients in stage III and one in stage IV died. Lethality was $3 \%$. Conclusions: Relapse of papillary carcinoma was associated with capsule involvement and lymphatic invasion. Tumor variants were not associated with relapse or mortality

Key words: Thyroid carcinoma, pathological variants, prognosis.

\section{Resumen}

Objetivo del estudio: Identificar la incidencia de las variantes del cáncer papilar de tiroides (CPT), factores de mal pronóstico asociados a variantes histológicas, comparar la recurrencia y estadio de la enfermedad según la variedad, e identificar pacientes de alto riesgo de recurrencia. Material y Método: Cohorte única longitudinal entre Enero de 1999 y Diciembre de 2008. La clasificación de los subtipos fue: papilar bien

*Recibido el 11 de Agosto de 2009 y aceptado para publicación el 19 de Enero de 2010.

Conflictos de intereses: no hay.

Correspondencia: Dr. Osvaldo Iribarren B.

Gabriela Mistral 2381, La Serena, Chile.

E-mail: oiribarren@123.cl 
diferenciado, variante folicular, esclerosante, sólido, células altas, células columnares, oncocítico, cribiforme, microcarcinoma, insular y pobremente diferenciado. Resultados: Incidencia: papilar bien diferenciado 40 casos $(63,5 \%)$, variante folicular 16 casos $(25,4 \%)$, esclerosante 4 casos, otros 3 casos. Hubo compromiso de la cápsula del tiroides en $6 / 27$ casos en estadio I, 4/9 casos en estadio II, 20/26 casos en estadio III y 1/1 caso en estadio IV, $\mathrm{p}<0,01$. Hubo 3 recurrencias ganglionares sin compromiso tumoral de la cápsula y 9 recurrencias con compromiso tumoral, $\mathrm{p}<0,05$. Hubo invasión linfática peritumoral en 12/27 en estadio I; 4/9 casos en estadio II, 21/ 26 casos en estadio III, y 1/1 en estadio IV, p $<0,05$. En 11/38 pacientes sin invasión linfática hubo recurrencia ganglionar y $27 / 38$ con invasión linfática hubo recurrencia, $p<0,01$. Fallecieron 2 casos en estadio III y 1 en estadio IV, p < 0,01. La letalidad fue 3,2\%. Conclusión: La recurrencia se asoció a estadio avanzado, compromiso de cápsula e infiltración linfática. No identificamos asociación entre recurrencia y variedades del CPT. La mortalidad se asoció a estadio avanzado. No identificamos asociación entre variante histológica y mortalidad, $\mathrm{p}=0,48$.

Palabras clave: Cáncer de tiroides, carcinoma papilar de tiroides, factores de pronóstico.

\section{Introducción}

El cáncer papilar (CPT) es la neoplasia maligna más frecuente del tiroides; se denomina carcinoma papilar al tumor epitelial maligno con diferenciación folicular, estructura papilar, y cambios nucleares caracterizados por gran tamaño, palidez, apariencia de vidrio esmerilado, borde irregular y pseudoinclusiones ${ }^{1}$. Este tumor tiene una sobrevida mayor al $90 \%$ en estadio I, II y III y las fallas de tratamiento se asocian a tumor avanzado, metástasis ganglionares e invasión histológica linfática y vascular ${ }^{2}$. Recientemente se han descrito subtipos de este cáncer que, además de tener una estructura histológica específica, tienen probablemente un significado pronóstico distinto al del carcinoma papilar clásico ${ }^{1,3}$. Los subtipos con mejor pronóstico corresponden a las formas papilares puras, variedad folicular ${ }^{3} \mathrm{y}$ encapsulado $^{4,5}$. Los CPT con peor pronóstico corresponden a la variedad esclerosante ${ }^{6,7}$, de células $\operatorname{altas}^{8-10}$ y no encapsulado ${ }^{9}$. Las variedades microcarcinoma y pobremente diferenciada, muestran resultados contradictorios. Algunos autores señalan un pronóstico intermedio $^{11-17}$ y otros señalan que presentan un mal pronóstico ${ }^{5,18}$. Las dos variantes más comunes corresponden a papilar puro y variante folicular ${ }^{19,20}$.

El objetivo de este estudio es:

1. Identificar la incidencia de las variantes del CPT.

2. Identificar factores de mal pronóstico asociados a variantes histológicas del CPT.

3. Comparar la recurrencia y estadio de la enfermedad, según la variedad histológica.

4. Identificar aquellos pacientes de alto riesgo de recurrencia que requieren vigilancia más intensiva.

\section{Material y Método}

Tipo de estudio: cohorte única longitudinal. Período del estudio: Enero de 1999-Diciembre de 2008.

\section{Criterios de inclusión:}

a. Pacientes con el diagnóstico de cáncer de tiroides, sin tratamiento previo, operados en el Servicio de Cirugía del Hospital Clínico San Pablo de Coquimbo y la Unidad de Oncología del Hospital San Juan de Dios de La Serena.

b. Pacientes con diagnóstico de cáncer papilar de tiroides en los que se revisó y reclasificó la variante histológica (coautores JAM, VZM) y que cuentan con estudio y registro histopatológico en el Servicio de Anatomía Patológica del Hospital Clínico San Pablo de Coquimbo, unidad que procesa y registra toda la información de la especialidad de los hospitales de la red asistencial pública de la IV Región, hasta el año 2008.

\section{Criterios de exclusión:}

a. Registros clínicos y/o histológicos incompletos. b. Casos detectados incidentalmente en autopsias.

c. Pacientes sometidos sólo a biopsias diagnósticas y/o que no fueron tratados con cirugía. No hubo pacientes que se excluyeran por los motivos $b$. y c.

La información relacionada con las operaciones, tratamientos complementarios y seguimiento fueron extraídas de las historias clínicas. En el período se identificaron 69 pacientes. En 6 casos hubo registros clínicos incompletos. En consecuencia, este estudio incluyó 63 pacientes.

En los fallecidos, su condición fue confirmada en el Registro Civil e Identificación.

\section{Clasificación de los tumores:}

Se hizo según los criterio TNM - OMS que considera $^{21}$ :

T: tamaño del tumor

$\mathrm{T}_{\mathrm{x}} \sin$ datos

$\mathrm{T}_{0}^{\mathrm{x}}$ no palpable

$\mathrm{T}_{1}<1 \mathrm{~cm}$. 
$\mathrm{T}_{2} 1-4 \mathrm{~cm}$.

$\mathrm{T}_{3}^{2}>4 \mathrm{~cm}$.

$\mathrm{T}_{4}^{3}$ cualquier tamaño con invasión extratiroídea

$N$ : estado de linfonodos

$\mathrm{N}_{\mathrm{x}}$ sin información

$\mathrm{N}_{0}^{\mathrm{x}}$ cervical no palpable

$\mathrm{N}_{1}$ adenopatía cervical ipsilateral

$\mathrm{N}_{2}$ adenopatía contralateral o bilateral

$\mathrm{N}_{3}^{2}$ adenopatías cervicales fijas

M: Metástasis

$M_{x}$ sin información

$\mathrm{M}_{0}^{\mathrm{x}}$ sin metástasis a distancia

$\mathrm{M}_{1}$ metástasis a distancias

Estadio de la enfermedad

Menor de 45 años

Estadio I: cualquier T, cualquier $\mathrm{N}, \mathrm{M}_{0}$

Estadio II: cualquier T, cualquier N, $\mathrm{M}_{1}$

Mayor de 45 años

Estadio I: $\mathrm{T}_{1}, \mathrm{~N}_{0}, \mathrm{M}_{0}$

Estadio II: $\mathrm{T}_{2}, \mathrm{~N}_{0}, \mathrm{M}_{0}, \mathrm{~T}_{3}, \mathrm{~N}_{0}, \mathrm{M}_{0}$

Estadio III: $\mathrm{T}_{4}, \mathrm{~N}_{0}, \mathrm{M}_{0}$, cualquier T, $\mathrm{N}_{1}, \mathrm{M}_{0}$

Estadio IV: cualquier T y cualquier $\mathrm{N}-\mathrm{M}_{1}$

Tratamiento quirúrgico: En presencia de diagnóstico pre e intraoperatorio de CPT, el tratamiento fue tiroidectomía total. Entre 1998 y 2005, en presencia de linfonodos histológicamente comprometidos en el estudio de biopsia por congelación, se efectuó una disección linfática selectiva lateral de los grupos II, III y IV, conservando la vena yugular interna. Entre 2005 y el cierre del estudio, se disecaron los linfonodos que aparecían ecográficamente sospechosos. (motivo de comunicación proxima). En presencia de linfonodos clínicamente metastáticos se efectuó disección radical de cuello modificada, que incluyó los grupos de linfonodos II, III, IV y V, con conservación de nervio accesorio y músculo esternocleido mastoideo ${ }^{22,23}$. En aquellos pacientes con diagnóstico confirmatorio post operatorio de cáncer, se completó la tiroidectomía en un segundo tiempo.

La clasificación de los subtipos histológicos de CPT incluyó las siguientes variantes: papilar bien diferenciado, variante folicular, esclerosante, sólido, células altas, células columnares, oncocítico, cribiforme, microcarcinoma, insular y pobremente diferenciado $^{24-29}$.

Tratamiento complementario: Luego de la tiroidectomía se efectuó tratamiento con radio yodo (80 - $150 \mathrm{~m} \mathrm{Ci} \mathrm{de} \mathrm{acuerdo} \mathrm{al} \mathrm{peso} \mathrm{del} \mathrm{paciente} \mathrm{y}$ extensión del tumor) y supresión - sustitución con tiroxina 100 - 150 microgramos/día ${ }^{21}$, según los valores $\mathrm{T}_{4}$ y TSH séricos.
El seguimiento se hizo con examen clínico, tiroglobulina plasmática (valor normal $<2$ microgr/L), nivel sérico de tiroxina y TSH, ecografía de cuello, radiografía de tórax y cintigrafía de cuerpo completo $^{25}$. No se incluyó la determinación de Ac anti TG en el seguimiento, ya que su incorporación en el arsenal de laboratorio clínico es de muy reciente data.

Para definir el hipoparatiroidismo se evaluaron los signos clínicos conocidos y la determinación de calcemia plasmática menor de $8,5 \mathrm{mg} / \%{ }^{26}$. Los pacientes fueron controlados 3 - 4 veces al año por 5 años y luego 1 vez al año.

\section{Definiciones}

a) Recurrencia local y/o regional: Enfermedad clínicamente detectable en el lecho del tiroides o linfonodos cervicales.

b) Metástasis a distancia: Enfermedad detectable clínica o radiológica en órganos distintos al lecho de la glándula o linfonodos cervicales.

Desenlace: se establecieron las siguientes opciones de cierre del estudio: Vivo sin enfermedad, vivo con metástasis locales y regionales, vivo con metástasis a distancia, vivo con metástasis en linfonodos y a distancia, fallecido por la enfermedad, fallecido por otra causa.

Análisis estadístico: los datos fueron recolectados en una base de datos EPI Info 3.5.

Se utilizó análisis univariable para comparar los grupos con distinto desenlace. Las variables continuas distribuidas normalmente se compararon utilizando la t de Student. Se utilizó la prueba estadística de chi cuadrado o la prueba exacta de Fischer para comparar variables categóricas. Se consideró significativo un valor de $\mathrm{p}=<0,05$.

\section{Resultados}

Se identificaron 51 mujeres y 12 varones. La edad media de las primeras fue 47,6 años \pm 16 y la edad media de los varones fue $42,4 \pm 18(\mathrm{p}=0,21)$. La incidencia de variantes histológicas del CPT fue: 40 casos con papilar bien diferenciado (63,5\%, 7 hombres y 33 mujeres), 16 casos con variante folicular de cáncer papilar $(25,4 \%, 2$ hombres y 14 mujeres), esclerosante en 1 hombre y 3 mujeres, pobremente diferenciado 1 (mujer), microcarcinoma 1 (mujer), células altas 1 (mujer), Tabla 1. La distribución por sexo no mostró asociación con las variedades histológicas, $(\mathrm{p}=0,15)$.

En estadio I hubo 25 mujeres $(39,6 \%)$ y 2 hombres $(3,2 \%)$; en estadio II hubo 8 mujeres $(12,6 \%)$ y 1 hombre $(1,6)$; en estadio III hubo 17 mujeres (27\%) y 9 hombres (14,3\%); en estadio IV hubo 1 mujer (1,6\%), Tabla 2. No identificamos asociación 
Tabla 1. Variedades histológicas cáncer papilar de tiroides

\begin{tabular}{lcrcrcr}
\hline Variedad & Hombres & \multicolumn{1}{c}{$\%$} & Mujeres & \% & Total & \% \\
\hline Papilar & 7 & 11,1 & 33 & 52,4 & 40 & 63,5 \\
Folicular & 2 & 3,2 & 14 & 22,2 & 16 & 25,4 \\
Esclerosante & 1 & 1,6 & 3 & 3,9 & 4 & 6,3 \\
Pobre Difer. & 0 & & 1 & 1,6 & 1 & 1,6 \\
Microcarcin. & 0 & & 1 & 1,6 & 1 & 1,6 \\
Cel. Altas & 0 & & 1 & 1,6 & 1 & 1,6 \\
Total & 10 & 15,8 & 53 & 84,1 & 63 & 100 \\
\hline
\end{tabular}

Tabla 2. Estadios clínicos. Cáncer papilar de tiroides

\begin{tabular}{ccrrrrr}
\hline Estadio & Hombres & \multicolumn{1}{c}{$\%$} & Mujeres & \% & Total & \% \\
\hline I & 2 & 3,2 & 25 & 39,6 & 27 & 42,8 \\
II & 1 & 1,6 & 8 & 12,6 & 9 & 14,2 \\
III & 9 & 14,3 & 17 & 27 & 26 & 41,3 \\
IV & 0 & & 1 & 1,6 & 1 & 1,6 \\
& 12 & 19,1 & 51 & 80,8 & 63 & 99,9 \\
\hline
\end{tabular}

Tabla 3. Estadios clínicos y variedades histológicas. Cáncer papilar de tiroides

\begin{tabular}{lccccc}
\hline Variedad & $\begin{array}{c}\text { Estadio } \\
\text { I }\end{array}$ & $\begin{array}{c}\text { Estadio } \\
\text { II }\end{array}$ & $\begin{array}{c}\text { Estadio } \\
\text { III }\end{array}$ & $\begin{array}{c}\text { Estadio } \\
\text { IV }\end{array}$ & Total \\
\hline Papilar & 16 & 7 & 15 & 1 & 39 \\
Folicular & 7 & 2 & 7 & 0 & 16 \\
Otras & 4 & 0 & 4 & 0 & 8 \\
Total & 27 & 9 & 26 & 1 & 63 \\
\hline
\end{tabular}

Tabla 4. Factores de riesgo de recurrencia. Cáncer papilar de tiroides

\begin{tabular}{lll}
\hline Factor de riesgo & \multicolumn{1}{c}{$\mathbf{p}$} & O.R \\
\hline Variedad & NS & \\
Invasión linfática & $<0,05$ & 9,7 \\
Infiltración cápsula & $<0,01$ & 3,9 \\
Estadio & N.S & 2,2 \\
Metástasis cervical & $<0,05$ & 9,4 \\
Edad: Mayor 45 años & N.S. & \\
\hline
\end{tabular}

NS: no significativo. O.R: Odds ratio. entre sexo de los pacientes y estadio clínico de la enfermedad $(\mathrm{p}=0,07)$.

La incidencia de las variantes histológicas según estadios clínicos fue: en estadio I, variedad papilar puro 16 casos $(59,3 \%)$, variedad folicular 7 casos $(25,9 \%)$, otras variedades en 4 casos $(14,9 \%)$; en estadio II papilar puro 7 casos $(77,8 \%)$, variedad folicular en 2 casos $(22,2 \%)$, en estadio III, variedad papilar puro en 15 casos $(57,7 \%)$, variedad folicular en 7 casos $(26,9 \%)$, otras variedades en 4 casos $(11,5 \%)$, en estadio IV 1 caso de papilar puro, Tabla 3. No identificamos asociación entre las variedades del CPT y estadio clínico de la enfermedad $(\mathrm{p}=0,9)$.

Se identificó compromiso de la cápsula del tiroides en $6 / 27$ casos en estadio I, 4/9 casos en estadio II, 20/26 casos en estadio III y $1 / 1$ caso en estadio IV, $\mathrm{p}<0,01$. Hubo 3 recurrencias ganglionares en pacientes que no tenían compromiso tumoral de la cápsula y 9 casos con recurrencia que tuvieron compromiso tumoral de la cápsula, $\mathrm{p}$ $<0,05$. Identificamos asociación entre compromiso de la cápsula de la glándula tiroides (carcinoma no encapsulado) y estadio clínico avanzado y, también con recurrencia de la enfermedad.

Se identificó invasión linfática tumoral en 12/27 casos en estadio I; 4/9 casos en estadio II, 21/ 26 casos en estadio III, y $1 / 1$ casos en estadio IV, $\mathrm{p}<0,05$.

En 51 casos $(80,9 \%)$ no hubo recurrencia hasta el cierre del estudio. En 12 casos $(19,1 \%)$ se presentó recurrencia ganglionar cervical. El intervalo libre hasta la aparición de la recurrencia fue 32,5 meses \pm 11 meses. Aquella se presentó en carcinoma bien diferenciado en 8 casos, variedad folicular 2 casos, esclerosante en 1 caso y células altas en un caso. Según estadio hubo recurrencia en 5 casos estadio I, 3 casos estadio II y 4 casos estadio III. No identificamos asociación entre las variantes histológicas y la recurrencia, $p=0,48$, ni entre las variantes y el estadio clínico de la enfermedad, $\mathrm{p}=0,09$, coeficiente de correlación $=0$.

En 1/38 pacientes sin invasión linfática hubo recurrencia ganglionar $\mathrm{y}$ 27/38 con invasión linfática hubo recurrencia, $p<0,01$, Tabla 4 . Identifica- 
mos asociación entre invasión linfática peritumoral y recurrencia de la enfermedad.

Evolucionaron sin complicaciones 51 casos $(81 \%)$. Las complicaciones del tratamiento quirúrgico fueron: hipoparatiroidismo transitorio en 6 casos $(9,5 \%)$, lesión de un nervio laríngeo recurrente en 4 casos $(6,3 \%)$, seroma o hematoma en 2 casos $(3,1 \%)$.

Al cierre del estudio, 48 pacientes están vivos y libres de enfermedad (76,2\%), 12 pacientes presentaron metástasis en linfonodos. La mortalidad fue de $4,8 \%$ y la letalidad fue de $3,2 \%$, en estadio III y IV, mayores de 45 años. Hubo 2 fallecidos por CPT y 1 fallecido por otra causa (empiema pleural). Dos fallecidos en estadio III y 1 caso en estadio IV, $\mathrm{p}<$ 0,01 ; Identificamos asociación entre estadio avanzado de la enfermedad y mortalidad. No identificamos asociación entre variante histológica y mortalidad, $\mathrm{p}=0,48$

\section{Discusión}

El CPT representa una diversidad de variantes morfológicas con un significado pronóstico poco claro. La mayoría son tumores duros, blanco grisáceos, de bordes irregulares y con evidente infiltración del parénquima del tiroides vecino, con tamaños que varían desde algunos milímetros hasta varios centímetros ${ }^{29}$. En Chile no se ha descrito la incidencia de las variedades de cáncer papilar, el pronóstico descrito en la literatura extranjera para cada una de ellas es contradictorio ${ }^{3-10}$.

En esta serie identificamos que las variedades más frecuentes de CPT son papilar puro y la variedad folicular, constituyendo ambos el $90 \%$ de los casos, (objetivo 1 del estudio).

En la búsqueda de factores de mal pronóstico de la enfermedad no encontramos asociación de aquel con el género de los pacientes ni con la variedad histológica de los tumores, En cambio, identificamos que el mal pronóstico se asocia significativamente al estadio avanzado de la enfermedad (estadio III y IV), infiltración tumoral de la cápsula del tiroides (variedad no encapsulados), e infiltración de los vasos linfáticos alrededor del tumor, hallazgos que son consistentes con publicaciones previas ${ }^{2,5,21,23,24,29}$ y que confirma el mal pronóstico de los CPT no encapsulados $^{9}$ (objetivo 2 ).

No encontramos asociación entre las variantes histológicas y recurrencia, ni entre esas variantes $\mathrm{y}$ estadio de la enfermedad o, entre las variantes y mortalidad, (objetivo 3). Esta ausencia de asociación a mal pronóstico se puede explicar en nuestra serie, por la baja incidencia de las variantes de CPT que tienen un conocido peor pronóstico como la variedad esclerosante ${ }^{6,7} \mathrm{y}$ de células altas ${ }^{8-10}$.
La sobrevida actuarial de $95 \%$ al cierre del seguimiento está dentro de lo descrito en publicaciones extranjeras ${ }^{6,21,30}$ y locales ${ }^{22,23}$, que señalan sobrevida global, luego de tratamiento asociado de cirugía y Yodo radiactivo $\left(\mathrm{I}^{131}\right)$, de hasta $95 \%$ y sobrevida hasta $100 \%$ en estadios I y II. La letalidad se limita a pacientes en estadio III y IV, mayores de 45 años,

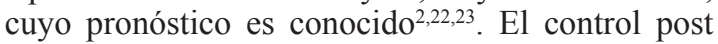
operatorio a intervalos más cortos con ecografía cervical seriada y determinación de antígenos y anticuerpos seriados en cada control clínico (tiroglobulina sérica y anticuerpos antiroglobulina), y una mejor educación de los pacientes con enfermedad avanzada y alto riesgo de falla de tratamiento, probablemente permitan un tratamiento de rescate y mejora de su sobrevida (objetivo 4).

La incidencia de resultados desfavorables asociadas a la técnica quirúrgica es similar a las descritas por autores nacionales ${ }^{23,24}$.

En resumen, aunque el seguimiento de esta casuística es reducido, los resultados sugieren que el riesgo de falla de tratamiento en el CPT se asocia a estadio clínico avanzado (tumor local avanzado y compromiso ganglionar de cuello), y condiciones histológicas desfavorables, como compromiso tumoral de la cápsula del tiroides e infiltración linfática peritumoral. Esta serie muestra que las variedades histológicas del CPT bien diferenciado y variante folicular tienen un pronóstico favorable a 10 años de seguimiento cuando son diagnosticadas y tratadas en estadio temprano, estadio I y estadio II.

\section{Referencias}

1. Chan J. Thyroid and Parathyroid. En Weidner, Cote, Suster, Weiss: Modern Surgical Pathology; 2003 Saunders, Philadelphia: 1661-1754.

2. Iribarren $\mathrm{O}$, Velasco N, Huidobro P, Núñez H, Villalón M, Madariaga J, y cols. Evolución y factores de pronóstico del cáncer diferenciado de tiroides. Rev Chil Cir 2009; 61: 136-141.

3. Furlan JC, Bedard YC, Rosen IB. Role of fine needle aspiration biopsy and frozen section in the management of papillary thyroid carcinoma subtypes. World J Surg 2004; 28: 880-885.

4. Bai Y, Kakudo K, Li Y, Liu Z, Ozaki T, Ito Y, Kihara M, et al. Subclassification of non solid type papillar thyroid carcinoma identification of high risk group in common type. Cancer Sci 2008; 99: 1908-1915.

5. Ito Y, Hirakawa M, Uruno T, Kihara M, Hiwashivama T, Takamura Y, et al. Prevalence and biological behaviour of variants of papillary carcinoma: experience at a single institute. Pathology 2008; 40: 617-622.

6. Chow SM, Chan JK, Law SC, Tang DL, Ho CM, Cheung WY, et al. Diffuse esclerosing variant papillary thyroid carcinoma. Clinical features and outcome. Eur 
J Surg Oncol 2003; 29 : 446-449.

7. Falvo L, Giacomelli L, D'Andrea V, Marzullo A, Guerreiro G, De Antonio E. Prognostic importance of sclerosing variant in papillary thyroid carcinoma. Ann Surg 2006; 72: 438-447.

8. Ylagan LR, Dehner LP, Huettner PC, Lu D. Columnar cell variant of papillary thyroid carcinoma. Report of a case with cytologic findings. Acta Cytol 2004; 48: 7377.

9. Das DK, Mallik MK, Sharma P, Sheikh ZA, Mathew PA, Sheikh M, et al. Papillary tyroid carcinoma and its variants in fine needle aspiration smears. A cytomorphologic study with special reference to tall cell variant. Acta Cytol 2004; 48: 325-336.

10. Leung AK, Chow SM, Law SC. Clinical features and outcome of the tall cell variant of papillary thyroid carcinoma. Laryngoscope 2008; 118: 32-38.

11. Fardella C, Jiménez M, González H, León A. Cruz F, Solar A, y cols. Características patológicas del microcarcinoma de tiroides. Revisión de 402 biopsias. Rev Med Chile 2005; 133: 1305-1310.

12. Hay ID, Hutchinson ME, González Losada T, McIver B, Reinalda M, Grant CS, et al. Papillary thyroid microcarcinoma: a study of 900 cases observed in a 60 years period. Surgery 2008; 144: 980-987.

13. Pakdaman MN, Rochon L, Gologan O, Tamilia M, Garfield N, Hier MP, et al. Incidence and histophatological behaviour of papillary microcarcinoma: study of 429 cases. Otolaryngol Head Neck Surg 2008; 139: 718-722.

14. Kim TY, Hong SJ, Kim JM, Gu Kim W, Gong G, Ryu JS, et al. Prognostic parameters for recurrence of papillary thyroid microcarcinoma. BMC Cancer 2008; 8 (296). http://www.biomedcentral.com/1471-2407/8/296, consultado Nov. 2009.

15. Besic N, Pilko G, Petric R, Hocevar M, Zgajnar J. Papillary thyroid microcarcinoma: prognostic factors and treatment. J Surg Oncol 2008; 97: 221-225.

16. Noguchi S, Yamashita H, Uchino S, Watanabe S. Papillary microcarcinoma. World J Surg 2008; 32: 747-753.

17. Hiltzic D, Carlson DL, Tuttle RM, Chuai S, Ishill N, Shaha A, et al. Poorly differenciated thyroid carcinomas defined on the basis of mitosis and necrosis: a clinicopathological study of 58 patients. Cancer 2006; 106: 1286-1295.

18. Miccoli P, Minuto MN, Ugolini C, Panicucci E, Massi
M, Berti P, et al. Papillary thyroid cancer: pathological parameters as prognostic factors in different classes of age. Otolaryngol Head Neck Surg 2008; 138: 200-203.

19. Burningham AR, Krishnan J, Davidson BJ, Ringel MD, Burman KD. Papillary and follicular variant of papillary carcinoma of the thyroid: initial presentation and response therapy. Otolaryngol Head Neck Surg 2005; 132: 840-844.

20. Uuksel O, Kuruhahvecioglu O, Ege B, Ekinci O, Aydin A, Poyraz A, et al. The relationship between papillary and follicular variant in papillary thyroid carcinoma. Endocr Regul 2008; 42: 29-33.

21. Lundgren CI, Hall P, Dickman PW, Zedenius J. Clinically significant prognostic factors for differenciated thyroid carcinoma. Cancer 2006; 106: 524-531.

22. Torres J. Cáncer diferenciado de tiroides. Rev Chil Cir 2000; 52: 21-218.

23. Pérez JA, Poblete MT, Ramírez R, Henning E, Uherek F, Salem C, y cols. Carcinoma diferenciado de tiroides. Rev Chil Cir 2000; 52: 585-592.

24. Chan J. Tumors of the thyroid and parathyroid glands. In Fletcher C: Diagnostic Histopathology of Tumors. Churchill Livingston 2007: 997-1017.

25. Ortiz S, Rodríguez JM, Parilla P, Sola J, Pérez D, Piñero A, et al. Papillary Thyroid Carcinoma. Arch Surg 2000, 135: 272-277.

26. Brinhurst R, Demay M, Krane S, Kronenberg HM. Metabolismo óseo y mineral en las personas sanas y enfermas. En Harrison: Principios de Medicina Interna 2006; $16^{\text {a }}$ Ed. Mc Graw Hill: 246-249.

27. Sherman S, Brierley J, Sperling M, Ain K, Bigos T, Cooper D, et al. Prospective multicenter study of thyroid carcinoma treatment. Cancer 1998; 83: 1012-1021.

28. Kakudo K, Tang W, Mori I, Nakamura Y, Miyauchi A. Papillary carcinoma of the thyroid in Japan: subclassification of common type and identification of low risk group. J Clin Pathol 2004; 57: 1041-1046.

29. Livolsi VA, Albores Saavedra J, Asa SL, Baloch ZW. Papillary carcinoma. En: De Lellelis R, Lloyd R, Heitz U, Eng Ch: Tumors of endocrine organs. Pathology \& Genetics. World Health Organization. Classification of Tumors, 2004; IARC Press, Lyon.

30. Lerch H, Schober O, Kuwert T, Saur HB. Survival of differentiated thyroid carcinoma studied in 500 patients. J Clin Oncol 1997; 15: 2067-2075. 